\title{
Centralidade municipal e interação estratégica na decisão de gastos públicos em saúde
}

\author{
Robson Fernandes Soares \\ Universidade Positivo (UP) / Programa de Mestrado e Doutorado em Administração \\ Curitiba - PR / Brasil \\ Ademir Clemente \\ Universidade Federal do Paraná (UFPR) / Programa de Pós-graduação em Contabilidade \\ Curitiba - PR / Brasil \\ Fátima de Souza Freire \\ Universidade de Brasilia (UnB)/ Programa de Pós-graduação em Ciências Contábeis \\ Brasília - DF / Brasil \\ Jorge Eduardo Scarpin \\ Universidade Federal do Paraná (UFPR) / Programa de Pós-graduação em Contabilidade \\ Curitiba - PR / Brasil
}

\begin{abstract}
Este artigo examina a influência que os municípios catarinenses exercem uns sobre outros nas decisões de gasto com saúde no ano de 2010. Partindo da teoria dos lugares centrais, tem-se como ponto focal a ideia de que há relações verticais entre municípios centrais e periféricos, principalmente em relação aos serviços mais específicos, como os hospitalares. Argumenta-se que esse tipo de interação vertical ocorre simultaneamente com a interação horizontal e que, quando a primeira é desconsiderada, pode mascarar esta última. Foi utilizado um modelo espacial autorregressivo para testar tal hipótese. Os resultados empíricos dão suporte a essa ideia, mostrando que municípios centrais apresentam maiores custos na subfunção hospitalar. Há também evidências de que, quando se considera a centralidade no modelo, o coeficiente de dependência espacial horizontal torna-se mais pronunciado.

Palavras-chave: interação governamental; dependência espacial; relação vertical; teoria dos lugares centrais; gasto público em saúde.

Centralidad municipal y la interacción estratégica en las decisions de los gastos en salud pública Este artículo examina cómo los municipios de Santa Catarina se influyen entre sí en las decisiones de gasto en salud, con base en el año 2010. Sobre la base de la teoría de los lugares centrales ha sido un
\end{abstract}

DOI: http://dx.doi.org/10.1590/0034-7612145797

(c) (i)

Artigo recebido em 8 fev. 2015 e aceito em 20 abr. 2016. 
punto focal para el análisis de la idea de que existe una relación vertical entre el centro y municipios periféricos, especialmente en relación con los servicios más específicos como los servicios hospitalarios. Se argumenta que este tipo de interacción vertical, se produce simultáneamente con la interacción horizontal, y cuando omiso puede ocultar esto. Se utilizó un modelo autorregresivo espacial para probar esta hipótesis. Los resultados empíricos apoyan esta idea, mostrando que los municipios centrales tienen incluso mayores costos en los servicios hospitalarios. La evidencia también muestra que, al considerar esta centralidad en la relación horizontal la dependencia especial es más pronunciada.

Palabras clave: interacción gobierno; dependencia espacial; relación vertical; teoría de los centrales lugares; el gasto público en salud.

\section{Municipal centrality and strategic interaction in deciding on public healthcare spending}

This article examines the influence that the municipalities of the state of Santa Catarina exerted on each other in deciding how much to spend on healthcare, in 2010. Using central place theory as a starting point, this paper focuses on the idea that there existed certain vertical relationships between central and peripheral municipalities, especially in more specific services such as those provided by hospitals. We argue that this type of vertical interaction occurs simultaneously with horizontal interaction and that, when the former is not considered, the latter may end up being concealed. We used a spatial self-regression model to test this hypothesis. The empirical results of the test support this idea, showing that the central municipalities have higher costs in terms of their sub-function of hospital services. We also found evidence that when the model takes centrality into account, then the horizontal spatial dependency coefficient becomes more pronounced.

KeyWords: government interaction; spatial dependence; vertical relationship; central place theory; public spending on healthcare.

\section{Introdução}

A interação estratégica entre entes públicos é um fenômeno que tem despertado a atenção da literatura econômica, inicialmente em estudos relacionados com a competição tributária para atrair empresas para suas jurisdições (Wilson, 1999), e em um segundo momento em relação aos padrões de relacionamento espacial que surgem também no comportamento dos gastos públicos (Brueckner, 2003).

Com relação aos gastos públicos, parte dos trabalhos se volta para o estudo da interação entre entes da mesma esfera (interação horizontal) e parte para a interação entre esferas diferentes (interação vertical). Abordagens que considerem a simultaneidade da ocorrência dos dois tipos de interação são escassas (Revelli, 2003). A exemplo de Aronsson, Lundberg e Wikström (2000), os trabalhos que consideram as duas modalidades de interação abordam a questão de hierarquia apenas de uma perspectiva de divisão política, tratando os entes pertencentes a uma mesma esfera como iguais. Até onde se pesquisou, apenas Werck, Heyndels

e Geys (2008) tratam da questão relativa à existência de hierarquia também dentro da perspectiva econômica. 
Os referidos autores procurando entender como os municípios belgas interagiam em relação às despesas com cultura, e dentro de uma perspectiva da teoria dos lugares centrais, verificaram a existência de um padrão de interação na relação entre municípios pequenos e municípios de grande porte, sugerindo a existência de uma hierarquia dentro da mesma esfera. No entanto, trabalharam com a função agregada, sem considerar como a especificidade do serviço influencia os padrões de interpendência horizontal e vertical.

O presente trabalho pretende ampliar o referido estudo, descendo a um nível menos agregado de subfunção e buscando entender em que medida essa especificidade do gasto está associada a um padrão diferente de interpendência das decisões de gasto em saúde. Mais especificamente se procura responder a seguinte pergunta de pesquisa: há diferença entre a influência da centralidade nos padrões de interdependência espacial de gastos públicos em saúde mais específicos e menos específicos para os municípios catarinenses no ano de 2010?

Para tanto são especificados alguns modelos espaciais em que é utilizado como variável dependente o gasto per capita, as funções de atenção básica (em que predominam serviços menos específicos/complexos), e outros nos quais a variável dependente é a assistência hospitalar e ambulatorial (em que predominam serviços mais específicos/complexos). Para cada conjunto de modelos é testada uma versão com e sem variável de centralidade, a fim de verificar como a consideração da centralidade interfere no padrão espacial verificado.

A compreensão dessa dinâmica se mostra particularmente importante nas discussões sobre políticas de transferências compensatórias relacionadas com os gastos com saúde, que tenham por objetivo reduzir as desigualdades regionais de investimento (Aronsson, Lundberg e Wikström, 2000), principalmente após a inclusão pela Lei no 141 de 2012 do perfil espacial como parâmetro a ser considerado nas transferências de recursos federais do fundo de saúde. Ainda, tal questão se mostra relevante na negociação dos termos de acordos regionais (consórcios), especificamente no que diz respeito à divisão das competências bem como às cláusulas de financiamento.

O texto está estruturado em cinco seções. Esta introdução, seguida do referencial teórico, em que se apresenta a evolução dos estudos sobre interdependência no gasto público, os principais contornos da teoria dos lugares centrais, utilizada como base para a análise, bem como um retrospecto do financiamento municipal de saúde no Brasil. A terceira e a quarta seções detalham a metodologia e os resultados da pesquisa. Na última seção são apresentadas as considerações finais.

\section{Referencial teórico}

\subsection{Interdependência na decisão de gasto público}

Partindo de uma análise das externalidades geradas pelos gastos públicos, uma primeira explicação teórica para a possibilidade de interação espacial no gasto público consiste na suposição de um possível efeito de transbordamento (spillover effect). Esse efeito surge quando 
os residentes de uma jurisdição consomem, de maneira direta ou indireta, os bens públicos de outra jurisdição (Isen, 2014). Nessa situação o gasto público de um município não atende apenas aos contribuintes daquela localidade, mas também cidadãos de outros lugares, que não são contribuintes do governo local.

Esse efeito de transbordamento pode ocorrer de duas formas. A primeira se dá quando os efeitos do gasto não estão limitados ao espaço geográfico do município que realizou o gasto, mas vão além, atingindo outros municípios. Exemplo disso são os investimentos para melhorar a qualidade da água de um rio que passa por duas cidades. Nesse caso, o município que investe na melhoria da água estará gerando um benefício não só para si próprio, mas também para o município vizinho, que receberá água de melhor qualidade sem ter participado financeiramente.

Na segunda forma não há extrapolação dos efeitos do gasto além do limite geográfico da localidade, mas o cidadão da cidade limítrofe se desloca até outro município para consumir um serviço ali oferecido (Solé-Ollé, 2006). Essa modalidade, mais comum nos serviços de saúde, ocorre quando uma pessoa se desloca até a cidade vizinha para obter uma especialidade não oferecida localmente, como serviços obstetrícios ou de tratamento intensivo. Nesse sentido quando uma localidade aumenta seus gastos, o vizinho tenderia teoricamente a investir menos, dando origem, portanto, a uma correlação espacial negativa.

Case, Rosen e Hines Jr. (1993) foram os primeiros a testar empiricamente o modelo de interação espacial. Eles verificaram que o aumento de uma unidade no gasto de um estado vizinho, além do esperado, fazia com que aumentasse 0,7 o gasto no estado considerado. Em seguida, Murdoch, Rahmatian e Thayer (1993), analisando os gastos municipais com lazer, e Kelejian e Robinson (1993), os gastos dos países com segurança, encontraram padrões semelhantes.

A partir desses resultados empíricos, Besley e Case (1995) propuseram um modelo informacional baseado na teoria de agência para explicar a possibilidade de correlação espacial positiva (yardstick competition). Segundo esse modelo, assume-se que o eleitor mediano (principal) possui uma dificuldade em avaliar os custos da prestação dos serviços públicos, portanto, de avaliar o desempenho de um político (agente) isoladamente.

Uma das formas de que o eleitor se vale para diferenciar os bons dos maus políticos consiste em comparar o valor gasto na prestação de serviços públicos por dois entes semelhantes, em uma espécie de benchmarking informacional. Os eleitores fazem uma comparação entre a quantidade de serviços que recebem e a quantidade de tributos que pagam (Brueckner, 2003). Nesse aspecto, o gestor público que investe percentualmente menos que seu vizinho poderá ser considerado um mau político. Tendo em mente esse comportamento do eleitor, o político que tem como objetivo reeleger-se ou manter seu partido no poder tenderá a imitar o gasto público do vizinho para não ser percebido como um mau político (Werck, Heyndels e Geys, 2008).

A maior parte dos trabalhos subsequentes encontrou evidências de correlação positiva, corroborando a segunda hipótese. Na área de gastos com saúde, Moscone e Knapp (2005), analisando a interação horizontal nos gastos com serviços de tratamento de doenças mentais 
na Inglaterra, verificaram que se situa entre 0,12 e 0,25, dependendo do modelo de análise. Costa-Font e Pons-Novell (2007), analisando os gastos na função saúde para os municípios espanhóis, encontraram relação entre 0,27 e 0,32.

Essa interação se mostrou sensível ao tipo de gastos em análise, apresentando variação entre 0,2 para serviços de internação até 0,7 para gastos com serviços primários no trabalho de Costa-Font e Moscone (2008) com municípios espanhóis. Já com relação ao aspecto temporal esse coeficiente apresentou variação entre 0,11 e 0,22 para diferentes anos no estudo de Moscone, Knapp e Tosetti (2007) com municípios ingleses.

\subsection{Teoria dos lugares centrais}

Nas abordagens empíricas de yardstick competition os trabalhos assumem como premissa que todos os municípios apresentam o mesmo padrão de interdependência em relação aos vizinhos. Revelli (2003) mostrou que essa correlação positiva poderia padecer de um viés de especificação por desconsiderar a interação vertical.

Dessa forma, embora a regressão mostre um efeito positivo de interdependência, o termo de erro ainda pode apresentar uma dependência espacial não modelada. Ao considerar esse efeito no modelo, o autor verificou que a correlação positiva foi suavizada com a inclusão de variável representativa da interação vertical. Isso significa que existe a possibilidade de coexistência de relação negativa e positiva dentro da mesma esfera.

Nesse sentido Werck, Heyndels e Geys (2008) verificaram que, ao se considerar o tamanho dos municípios, é possível observar uma assimetria dos gastos dos menores em relação aos maiores, o que também indica um efeito vertical não modelado. No referido trabalho, os municípios menores apresentaram uma interdependência positiva em relação aos municípios menores e negativa em relação aos grandes centros. A explicação para essa existência de dois padrões dentro da mesma esfera encontra suporte na teoria dos lugares centrais.

A origem dessa teoria é atribuída aos trabalhos de Christaller (1966) e Lösch (1954), que propuseram um modelo de oferta de bens e serviços para uma demanda distribuída espacialmente. Segundo essa teoria, dados os custos elevados de determinados empreendimentos, sua viabilidade somente é possível se houver um número mínimo de usuários (Werck, Heyndels e Geys, 2008). Dessa forma, se o número mínimo de usuários que viabiliza o negócio for maior que a população de uma cidade, torna-se economicamente inviável a manutenção de determinado empreendimento. Isso implica que cidades com populações pequenas podem não ter determinados serviços e necessitem recorrer aos seus vizinhos.

Em Santa Catarina, por exemplo, há apenas 166 cidades que possuem leitos de obstetrícia, o que faz com que os habitantes dos outros 127 municípios tenham de se deslocar a um desses centros para ter acesso ao serviço. Com relação às unidades de tratamento intensivo, existem apenas 22 municípios que dispõem de tal atendimento, o que faz com que os outros 271 tenham de remover seus pacientes mais graves para uma dessas unidades. 
Segundo Christaller (1966), os grandes centros (metrópoles) oferecem todos os bens e serviços e à medida que a localidade se torna mais periférica há uma menor variedade de bens e serviços ofertados. Isso gera um problema de alocação espacial, em que alguns consumidores das localidades periféricas simplesmente não têm acesso a determinados bens e serviços pelo fato de ser muito caro para eles o custo de deslocamento até a localidade central, o que gera uma exclusão espacial do consumo (Nakamura, 2014). Isso cria uma hierarquia entre as cidades, uma vez que as cidades pequenas são dependentes em relação às de médio porte e essas em relação às de grande porte no fornecimento de determinados bens e serviços.

Outra explicação para a existência de serviços exclusivos de grandes centros, trazido por Christaller (1966) e não explorado no trabalho de Werck, Heyndels e Geys (2008), diz respeito à especificidade do serviço. Quanto mais comum for o serviço, maior será a demanda e mais provável sua viabilidade em pequenas cidades; por outro lado, quanto mais específica for a atividade, menor sua demanda e mais difícil sua viabilidade em centros de pequeno porte. Isso explica por que serviços de postos de saúde são ofertados em quase todos os municípios ao passo que determinados tipos de cirurgias são encontradas apenas em grandes centros.

Tendo por base o trabalho de Costa-Font e Moscone (2008), que verificou ser a dependência espacial relacionada com o tipo de gasto, e tomando por base a teoria dos lugares centrais e a relação entre centralidade e especificidade apontada por Christaller (1966), tem-se como hipótese que em gastos relacionados com serviços mais específicos haja uma relação positiva entre centralidade e gasto público per capita, ao passo que em serviços mais comuns haja uma relação negativa. Na demanda por serviços comuns, oferecidos em todos os municípios, não haveria a migração intermunicipal e existiria a predominância de ganhos de escala em municípios centrais, fazendo com que o custo por habitante da prestação dos serviços seja menor nestes. Por outro lado, nos serviços complexos que são oferecidos em apenas alguns municípios, haveria um fluxo inter-regional, inflacionando o custo per capita dos municípios centrais.

Da perspectiva orçamentária de saúde, os gastos na subfunção de atenção básica são direcionados a recursos de menor especificidade, ao passo que os gastos na subfunção de assistência hospitalar e ambulatorial apresentam direcionamento para serviços de maior especificidade. Dessa forma, tem-se as seguintes hipóteses:

(H1): A centralidade está negativamente associada ao gasto público em Atenção Básica;

(H2): A centralidade está positivamente associada ao gasto público per capita em Assistência Hospitalar e Ambulatorial.

Adicionalmente, procura-se testar a hipótese de coexistência de efeito vertical e horizontal proposta por Revelli (2003). Dessa perspectiva, espera-se que na relação horizontal entre municípios centrais e periféricos haja uma correlação negativa, ao passo que na relação horizontal haja uma correlação positiva em decorrência da yardstick competition. Assim, no modelo que não considera a interação vertical haveria uma subestimação do coeficiente de interação horizontal. Dessa forma, a adição de uma variável representativa dessa relação vertical negativa faria com que o coeficiente positivo fosse mais bem estimado. Disso resulta a seguinte hipótese:

(H3): O coeficiente de dependência espacial aumenta com a inclusão da variável de centralidade. 


\subsection{0 orçamento de saúde no Brasil e o gasto municipal}

Conforme Ugá (2012), a experiência internacional consagra duas formas de financiamento dos gastos em saúde. A primeira é um financiamento prévio (ex ante), em que a alocação orçamentária tem por base um percentual específico do orçamento. A segunda é um financiamento posterior (ex post), no qual o total gasto se dá com base no volume prestado de serviços hospitalares.

O sistema de saúde brasileiro adotou uma forma mista de alocação orçamentária aos municípios, no qual parte do orçamento lhes é transferido principalmente por repasses diretos ao Fundo Municipal de Saúde, outra parte é paga diretamente aos estabelecimentos prestadores de serviço conforme sua produção e obedecendo aos valores da tabela padronizada de procedimentos do Sistema Único de Saúde (SUS), e uma terceira fonte de recursos provém da aplicação direta municipal de um percentual mínimo de seus recursos próprios.

Esse sistema de financiamento encontra-se normatizado principalmente no art. 198 da Constituição Federal de 1988, nas Leis Federais nos 8.080/1990, 8.142/1990 e 141/2012, Decreto no 7.508/2011, nas Normas de Operacionalização Operacional de Assistência à Saúde - Noas/SUS nos 01/1996 e 01/2002, bem como nas Portarias do Ministério da Saúde GM/ MS nos 399/2006 e 1.091/2006. É possível encontrar uma revisão da evolução legislativa do financiamento na cartilha do Conselho Nacional de Secretários de Saúde (Conass, 2011).

A parte transferida aos municípios deve obedecer ao art. 35 da Lei no 8.080/1990, que estabelece critérios a serem observados na distribuição dos recursos. Para tanto foram editadas as Noas/SUS nํㅗ 0119/96 e 01/2002, que definem para os municípios cadastrados um valor fixo per capita que é repassado para atender gastos com assistência básica de saúde da população e valores variáveis que são repassados de acordo com a adesão a programas específicos de governo, como saúde família. Há também uma parcela variável repassada para atendimentos de média e alta complexidade que se dá em forma de ressarcimento dos serviços prestados pelos estabelecimentos de saúde municipais e está sujeita a um teto.

Esse modelo de ressarcimento, conforme Ellis e McGuire (1996), acaba gerando incentivos que distorcem a qualidade do atendimento, pois, como o pagamento se dá por procedimentos, há uma tendência à submissão do paciente a mais procedimentos do que o necessário para angariar mais fundos, e evitar procedimentos que custem mais do que o previsto na tabela SUS.

Já em relação à parcela aplicada diretamente, deve haver uma observância à Lei no 141/2012, que obriga a aplicação mínima de 15\% do somatório dos impostos municipais e das transferências recebidas do Fundo de Participação dos Municípios/Estados (FPM e FPE) em gastos com saúde. Conforme Vazquez (2011), o estabelecimento desse patamar mínimo de investimento resultou em uma ampliação da participação dos governos subnacionais na oferta e atendimento básico de saúde.

Essa demanda por gastos diretos dos municípios foi reforçada pela exigência de contrapartidas municipais para fazer frente aos custos dos programas aderidos, que ao longo dos últimos anos não tiveram seus repasses adequadamente corrigidos. Como consequência, houve um aumento médio de $83 \%$ no gasto per capita financiado com recursos municipais de 
2000 a 2006 (Vazquez, 2011). Esse cenário tem demostrado uma tendência de ampliação da responsabilidade do município na execução e financiamento da política de saúde já apontada por Mendes e Santos (2000), e observada na aplicação média de recursos próprios na ordem de $19,43 \%$ para 2010 em Santa Catarina.

Para os fins do presente trabalho, não se utilizou de variáveis dependentes que considerassem a fonte de financiamento do recurso, uma vez que a hipótese de yardstick competition está baseada na percepção comparativa do eleitor em face do gasto dos municípios vizinhos de maneira agregada. A consideração da despesa por fonte seria uma sofisticação do modelo, que pressuporia que o eleitor seria capaz de discriminar a origem do recurso e então avaliar o esforço fiscal próprio.

Contudo, mesmo não havendo a segregação da despesa por fonte, houve o reconhecimento da dependência financeira de outros entes na decisão de gastos públicos agregados. Essa questão foi tratada no modelo mediante uma variável que mediu a proporção de receitas de transferências correntes em relação a receitas correntes. Essa também é uma medida de interdependência vertical entre entes de esferas diferentes.

\section{Metodologia da pesquisa}

\subsection{População e coleta de dados}

O trabalho objetivou analisar o padrão de gastos em saúde de municípios vizinhos, para isso sendo necessário utilizar técnicas de econometria espacial. Para essas técnicas, a ausência de dados se torna um problema mais delicado, pois a desconsideração de uma unidade afeta também a mensuração das unidades do entorno. Isso faz com que o grau de cobertura seja uma questão importante a se considerar na seleção da amostra.

Dessa forma, procurando trabalhar com o mínimo de dados ausentes para as variáveis dependentes, optou-se por selecionar municípios de um estado que houvesse apresentado 100\% de cobertura das informações financeiras de gastos com saúde. Consultando a base de dados do Finbra para o ano de 2010, verificou-se que Santa Catarina foi o estado que atendeu a tal critério, não apresentando dados ausentes.

\subsection{Variáveis}

Buscou-se identificar como as decisões de investimento em saúde de um município são afetadas por seu grau de centralidade. Para tanto foram selecionados como variáveis dependentes os gastos per capita na função saúde, assim como em estudos anteriores, bem como os gastos nas subfunções de Atenção Básica e Assistência Hospitalar e Ambulatorial.

A centralidade é considerada no presente estudo como Proxy da interação vertical. Como constructo de centralidade utilizou-se a classificação do IBGE - que tem como base os 
trabalhos de Christaller (1966). Nela os municípios são classificados de acordo com a posição hierárquica que ocupam na rede urbana.

Dessa forma os municípios podem ser classificados desde metrópoles, que possuem extensa área de influência, até centros locais, cuja centralidade não ultrapassa os limites de sua jurisdição. Conforme tal classificação, Santa Catarina apresenta 247 municípios classificados como centros locais, que não possuem centralidade que extrapole os limites territoriais do município, e 46 municípios classificados em níveis superiores de centralidade, e que, portanto, exercem influência sobre um ou mais municípios vizinhos.

Para capturar o efeito da centralidade sobre a decisão de gastos com saúde utilizou-se uma variável binária que assume 0 se o município é classificado como centro local, e 1 se apresenta nível de centralidade maior. As demais variáveis da pesquisa são relacionadas no quadro 1.

Quadro 1

Variáveis da pesquisa

\begin{tabular}{|c|c|c|c|c|}
\hline \multicolumn{5}{|c|}{ Variáveis dependentes } \\
\hline Variável & \multicolumn{3}{|l|}{ Descrição } & $\begin{array}{c}\text { Base de } \\
\text { dados }\end{array}$ \\
\hline Saúde & \multicolumn{3}{|l|}{ Gasto per capita na função Saúde } & Finbra \\
\hline Atenção Básica & \multicolumn{3}{|l|}{ Gasto per capita na subfunção de Atenção Básica } & Finbra \\
\hline $\begin{array}{l}\text { Assistência } \\
\text { Hospitalar }\end{array}$ & \multicolumn{3}{|c|}{ Gasto per capita na subfunção Assistência Hospitalar e Ambulatorial } & Finbra \\
\hline \multicolumn{5}{|c|}{ Variável independente } \\
\hline Variável & \multicolumn{3}{|l|}{ Descrição } & $\begin{array}{l}\text { Base de } \\
\text { dados }\end{array}$ \\
\hline & \multicolumn{3}{|c|}{$\begin{array}{l}\text { Dummy que assume } 0 \text { se o município é classificado como centro local, e } 1 \text { se apresentar } \\
\text { outro nível de centralidade }\end{array}$} & IBGE \\
\hline \multicolumn{5}{|c|}{ Variáveis de controle } \\
\hline Variável & Descrição & $\begin{array}{c}\text { Sinal } \\
\text { Esperado }\end{array}$ & $\begin{array}{l}\text { Autor de } \\
\text { Referência }\end{array}$ & $\begin{array}{l}\text { Base de } \\
\text { dados }\end{array}$ \\
\hline$P I B$ & Produto interno bruto per capita & Positivo & Freeman (2003) & Data-SUS \\
\hline Idosos & Percentual da população com mais de 60 anos & Positivo & Di Matteo (2005) & Data-SUS \\
\hline Crianças & Percentual da população com menos de quatro anos & Positivo & $\begin{array}{l}\text { Prieto e Lago- } \\
\text { Peñas (2012) }\end{array}$ & Data-SUS \\
\hline Gênero & Razão entre mulheres e homens na população & Positivo & $\begin{array}{l}\text { Moscone e Knapp } \\
\text { (2005) }\end{array}$ & Data-SUS \\
\hline Médicos & Número de profissionais de saúde por mil habitantes & Positivo & $\begin{array}{l}\text { Costa-Font e } \\
\text { Moscone (2008) }\end{array}$ & Data-SUS \\
\hline Leitos & Número de leitos por mil habitantes & Negativo & $\begin{array}{l}\text { Giannoni e Hitiris } \\
\text { (2002) }\end{array}$ & Ipea-GEO \\
\hline Plan. Saúde & Percentual da população coberta por planos de saúde & Negativo & $\begin{array}{l}\text { Novaes e Mattos } \\
(2010)\end{array}$ & Data-SUS \\
\hline
\end{tabular}




\begin{tabular}{|c|c|c|c|c|}
\hline Esgotamento & $\begin{array}{l}\text { Percentual de pessoas com esgotamento sanitário } \\
\text { considerado inadequado }\end{array}$ & Positivo & $\begin{array}{l}\text { Novaes e Mattos } \\
(2010)\end{array}$ & Data-SUS \\
\hline Mortalidade & Taxa de mortalidade bruta & Positivo & $\begin{array}{l}\text { Moscone, Knapp e } \\
\text { Tosetti (2007) }\end{array}$ & Ipea-GEO \\
\hline D Governador & Dummy para mesmo partido do governador & Positivo & $\begin{array}{l}\text { Novaes e Mattos } \\
\text { (2010) }\end{array}$ & TRE-SC \\
\hline D Presidente & Dummy para mesmo partido do presidente da república & Positivo & $\begin{array}{l}\text { Novaes e Mattos } \\
(2010)\end{array}$ & TRE-SC \\
\hline Dependência & Razão entre transferências correntes e receitas correntes & Positivo & Exploratória & Finbra \\
\hline$P R ; R S ; A R G$ & $\begin{array}{l}\text { Dummies para municípios de divisa com Paraná, Rio } \\
\text { Grande do Sul e Argentina }\end{array}$ & Indefinido & Almeida (2012). & - \\
\hline
\end{tabular}

Fonte: Elaboração própria.

As decisões de gasto em saúde são influenciadas por características demográficas e econômicas da população, bem como estruturas políticas e de infraestrutura do município (Moscone, Knapp e Tosetti, 2007). Dentro dessa linha foram selecionadas as principais variáveis trazidas pela literatura.

O fator renda é tido pela literatura como o principal determinante dos gastos públicos em saúde. Há uma série de trabalhos que demonstram a existência de relação positiva entre a renda familiar e os gastos governamentais nessa função (Freeman, 2003). No Brasil, Sousa (2012) e Boulding e Brown (2014) encontraram evidências no mesmo sentido. Para representar tal variável foi selecionado o Produto Interno Bruto per capita.

A variável representativa da estrutura demográfica tem sido principalmente a estrutura etária da população. Os estudos procuram identificar segmentos populacionais que demandam por maiores gastos com saúde. Dentro dessa linha, Di Matteo (2005), analisando dados dos estados canadenses e americanos, verificou que, quanto maior a população idosa dos estados, tanto maior era o gasto com saúde, e que com o aumento da idade também aumentam os gastos. Ainda nessa linha, Prieto e Lago-Peñas (2012) encontraram evidências de maiores gastos com saúde associados à proporção de crianças com menos de quatro anos.

Outras duas variáveis associadas a características de segmentos populacionais que demandam maiores gastos com saúde são gênero e taxa de mortalidade (Moscone e Knapp, 2005; Moscone, Knapp e Tosetti, 2007; Novaes e Mattos, 2010). Com relação ao gênero, a explicação reside nos gastos adicionais com serviços de ginecologia e obstetrícia que o segmento feminino demanda. Quanto à mortalidade, quanto maiores seus níveis, maior a pressão social para que o Estado realize gastos maiores com saúde.

Com relação à estrutura de prestação do serviço, Giannoni e Hitiris (2002) verificaram que o governo italiano apresentava um gasto per capita menor nas regiões em que a quantidade de leitos per capita era maior. Essa relação é atribuída ao ganho de escala obtido. Por outro lado, quanto maior a proporção de pessoal de saúde per capita, tanto maiores eram os gastos. A mesma relação foi encontrada por Costa-Font e Pons-Novell (2007) ao analisarem os estados espanhóis. 
Novaes e Mattos (2010), no contexto brasileiro, associaram os gastos com saúde à cobertura da rede de esgoto, como forma de controlar os gastos de saúde como possíveis reações às condições fitossanitárias dos municípios. Essa variável não é comumente encontrada em estudos internacionais, que abordam normalmente países desenvolvidos, mas para o caso do Brasil a inclusão de tal variável é relevante e se mostrou significativa, apresentando relação positiva com o gasto público com saúde. Também se espera que, quanto maior a cobertura de planos de saúde, menor a necessidade de gastos públicos para atender a população, esperando-se, portanto, uma relação inversa entre ambos (Novaes e Mattos, 2010).

Com relação à ideologia partidária, há uma tendência de que os partidos de esquerda invistam mais em saúde e os partidos de direita deixem tal tarefa para a iniciativa privada (Costa-Font e Moscone, 2008). Contudo, os resultados empíricos são mistos. Nesse sentido, Le Maux, Rocaboy e Goodspeed (2011) verificaram que na França os partidos de esquerda investem mais em gastos sociais. Por outro lado, Potrafke (2009), analisando os gastos sociais dos países pertencentes à Organização para a Cooperação e Desenvolvimento Econômico (OCDE), e Sousa (2012), analisando os gastos em saúde dos municípios pernambucanos, não encontraram relação entre os gastos e a orientação político-partidária. No presente trabalho, tendo 2010 como de análise, o partido da presidente é de esquerda e o do governador, de direita.

Também se procurou analisar de forma exploratória como a dependência de transferências influencia no comportamento de gasto.

Ainda foram utilizadas variáveis dummy para identificar municípios que fazem fronteira com outros estados, conforme proposto por Almeida (2012). Isso porque é preciso diferenciar os municípios do interior, que possuem todos os vizinhos considerados na matriz, dos municípios de divisa, que só serão considerados como vizinhos os municípios catarinenses.

\subsection{Técnica de análise e especificação do modelo}

Para a análise dos dados foi utilizada, em um primeiro momento, a estatística $I$ de Moran para identificar a existência de padrões espaciais de interação. O I de Moran é um teste de autocorrelação espacial entre observações que têm um grau de vizinhança especificado pela matriz de pesos espaciais (W). A interpretação é similar à do teste de correlação de Pearson: 0 indica inexistência de autocorrelação espacial, 1 indica autocorrelação positiva perfeita, e -1, autocorrelação negativa perfeita. O índice é formalmente definido como:

$$
I=\frac{n}{S_{0}} \frac{\sum_{i} \sum_{j} w_{i j} z_{i} z_{j}}{\sum_{i=1}^{n} z_{1}^{2}}
$$

O arranjo espacial dos dados é incorporado ao modelo por meio de uma matriz de ponderação espacial $(W)$ em que cada célula representa a distância entre pares de dados de duas regiões. Pressupõe-se que regiões mais próximas entre si interagem mais do que regiões 
mais afastadas (Almeida, 2012). No presente trabalho foram utilizadas matrizes baseadas nas distâncias geográficas e na contiguidade, conforme o quadro 2.

Quadro 2

Matizes de distância espacial

\begin{tabular}{|c|c|c|}
\hline W & Especificação & Descrição \\
\hline Contiguidade & $\begin{array}{l}w_{i j}(k) \\
=\left\{\begin{array}{c}1-\text { se } \text { i e } j \text { são contiguos } \\
0-\text { se } \text { i } \text { e } j \text { não são contíguos }\end{array}\right.\end{array}$ & $\begin{array}{l}\text { Quando as regiões são contíguas a célula entre elas apresenta } \\
\text { valor 1, caso contrário, apresenta 0. Quando para definição da fronteira } \\
\text { se utiliza apenas o critério de extensão territorial, elas são classificadas } \\
\text { como Rook, quando se considera também as divisas em relação aos } \\
\text { vértices (para todos os lados), é classificada com Queen. Ainda, em } \\
\text { relação à ordem, podem ser considerados como vizinhos: os vizinhos } \\
\text { diretos (de 1a ordem); os vizinhos dos vizinhos (2ª ordem); os vizinhos } \\
\text { dos vizinhos dos vizinhos (3a ordem) e assim por diante. }\end{array}$ \\
\hline $\begin{array}{l}\text { Distância } \\
\text { geográfica }\end{array}$ & $w_{i j}(k)=\left\{\begin{array}{l}1-\text { se } d_{i j} \geq d_{c} \\
0-\text { se } d_{i j}<d_{c}\end{array}\right.$ & $\begin{array}{l}\text { Quando as regiões se encontram até determinada distância de } \\
\text { corte, a matriz assume 1, caracterizando a vizinhança, se não, assume } \\
\text { 0. É possível construir tantas matrizes, quantos forem os critérios de } \\
\text { corte estabelecidos. Esse critério pode considerar a distância em milhas } \\
\text { ou quilômetros, bem como considerar a curvatura da terra. }\end{array}$ \\
\hline
\end{tabular}

Fonte: Elaboração própria.

O cálculo do coeficiente depende da matriz W utilizada; portanto, para diminuir a arbitrariedade é utilizado procedimento similar ao proposto por Baumont (2004), em que se estima o modelo clássico de regressão, testa-se o modelo para autocorrelação com base no $I$ de Moran para várias matrizes W e seleciona-se a matriz que tenha gerado o maior índice de Moran significativo.

No presente trabalho, uma autocorrelação positiva significa que, quando um município aumenta seus gastos em saúde, o vizinho, em resposta, também aumentará seu nível de gasto. Ao contrário, a autocorrelação negativa indica que, quando um município gasta mais em saúde, o vizinho tenderá a gastar menos.

Em seguida, a relação espacial foi modelada a fim de melhor compreender a dependência espacial e analisar o papel da centralidade. Para tanto, foi utilizado o modelo espacial autorregressivo para estimar a função de reação fiscal dos gastos públicos em saúde. Foram especificados dois modelos básicos: o primeiro sem considerar a variável de centralidade e o segundo incluindo a métrica de centralidade, conforme as equações a seguir.

$$
\begin{gathered}
s=\rho W s+\beta_{j} X_{j}+\varepsilon \\
s=\rho W s+\beta_{j} X_{j}+C+\varepsilon
\end{gathered}
$$

Nesses dois modelos, $s$ representa o gasto público em saúde, conforme os três constructos de decisão de investimento em saúde; $W$ representa a matriz de distâncias, de ordem 293x293; $X_{j}$ representa uma matriz contendo o termo de intercepto e o conjunto de variáveis de controle, $C$ é a dummy de centralidade; e $\varepsilon$ diz respeito ao termo estocástico. Nesse modelo, 
$\rho$ (roh) é o parâmetro autorregressivo. Quando é $\rho$ positivo, há indicação de autocorrelação positiva; quando $\rho$ é negativo, a interpretação é de existência de autocorrelação negativa.

Cabe destacar que a estimação desse modelo espacial não pode ser feita por mínimos quadrados ordinários, pois, conforme Revelli (2005), isso traz um viés de especificação aos parâmetros $\beta$. Em função disso, a estimação é feita pelo método de máxima verossimilhança, com o auxílio do Software GeoDa (C) 1.6.2.

\section{Resultados}

\subsection{Estatísticas descritivas}

Com intuito de caracterizar os municípios, são apresentados as médias, desvios padrões, valores mínimos e máximos das variáveis utilizadas na regressão tomando como base o período de 2010. Foram calculados os valores médios das variáveis para os municípios que possuem centralidade e para os que não possuem, e efetuou-se o teste de diferença de médias. Os resultados são apresentados na tabela 1.

Tabela 1

Estatísticas descritivas

\begin{tabular}{|lrrrrrrrr|}
\hline & Média & $\begin{array}{c}\text { Desvio- } \\
\text { padrão }\end{array}$ & $\begin{array}{c}\text { Coef. de } \\
\text { variação }\end{array}$ & Mínimo & Máximo & $\begin{array}{r}\text { Médias não } \\
\text { centrais }\end{array}$ & $\begin{array}{r}\text { Médias } \\
\text { centrais }\end{array}$ & Anova \\
\hline Saúde & 454.87 & 172.98 & 0.38 & 173.50 & 1315.36 & 470.36 & 373.80 & 0.00 \\
Aten. Básica & 398.22 & 185.88 & 0.47 & 0.00 & 1275.35 & 423.74 & 264.68 & 0.00 \\
Ass. Hospitalar & 34.81 & 74.12 & 2.13 & 0.00 & 580.84 & 27.17 & 74.82 & 0.00 \\
PIB & 18527.47 & 9039.47 & 0.49 & 8092.85 & 93592.80 & 18631.46 & 17983.16 & 0.65 \\
Idosos & 12.33 & 2.49 & 0.20 & 6.60 & 19.60 & 12.62 & 10.82 & 0.00 \\
Crianças & 6.36 & 0.93 & 0.15 & 3.70 & 9.40 & 6.33 & 6.53 & 0.16 \\
Gênero & 102.88 & 6.24 & 0.06 & 90.60 & 174.10 & 103.78 & 98.19 & 0.00 \\
Médicos & 9,66 & 3,02 & 0,31 & 3,26 & 31,03 & 9,3 & 11,6 & 0.00 \\
Leitos & 0,57 & 1.024 & 1.79 & 0.00 & 6,5 & 0.62 & 0.26 & 0.00 \\
Plan. Saúde & 12.17 & 12.09 & 0.99 & 0.87 & 89.90 & 10.43 & 21.31 & 0.00 \\
Esgotamento & 13.15 & 14.09 & 1.07 & 0.00 & 63.20 & 14.63 & 5.44 & 0.00 \\
Mortalidade & 5.83 & 1.36 & 0.23 & 1.62 & 10.73 & 5.84 & 5.76 & 0.70 \\
Dependência & 0.85 & 0.12 & 0.14 & 0.31 & 1.08 & 0.87 & 0.73 & 0.00 \\
D Governador & 0.52 & 0.50 & 0.96 & 0.00 & 1.00 & 0.53 & 0.45 & 0.28 \\
D Presidente & 0.12 & 0.32 & 2.72 & 0.00 & 1.00 & 0.11 & 0.17 & 0.24 \\
Centralidade & 0.16 & 0.37 & 2.29 & 0.00 & 1.00 & & & \\
\hline
\end{tabular}

Fonte: Elaboração própria a partir dos dados coletados. 
O gasto médio anual na função saúde varia consideravelmente de município para município, apresentando valores que vão de $\mathrm{R} \$ 173,50$ anuais gastos por habitante até $\mathrm{R} \$ 1.315,36$.

Essa variação é mais acentuada em relação aos gastos com a função de Assistência Hospitalar e Ambulatorial, que apresentam um coeficiente de variação de 2,13 . Isso se dá porque existem dois perfis distintos de investimento nessa subfunção, um de municípios centrais que apresentam gastos médios de $\mathrm{R} \$ 74,82$ e outro de municípios periféricos que apresentam gasto médio de $\mathrm{R} \$ 27,17$. Essa relação é condizente com o esperado, uma vez que teoricamente se prevê que os gastos hospitalares das localidades centrais tendem a ser mais elevados em razão do atendimento de populações de outras localidades.

Com relação aos gastos com atenção básica, a relação é inversa, havendo um menor gasto por habitante nas localidades centrais. Isso se dá porque esse é um serviço de menor complexidade, ofertado por todas as localidades. Dessa forma, nessa modalidade de gasto predomina o ganho de escala com o aumento da população, o que também é condizente com a teoria.

Com relação ao gasto agregado, considerando a função saúde como um todo, também se verifica a predominância de um comportamento de ganho de escala, uma vez que a maior parte desses gastos é decorrente de gastos com atenção básica.

Ainda, é possível verificar que, ao se considerar a centralidade municipal, alguns fatores determinantes dos gastos com saúde apresentam valores médios significativamente diferentes nos municípios centrais. Isso reforça a necessidade de utilizá-los como variáveis de controle na regressão, para que se possa capturar a influência da centralidade líquida dessas variações próprias das características municipais.

Exemplo disso é a proporção de idosos, que nas cidades centrais consiste em média em $10,82 \%$ da população, ao passo que nas cidades periféricas, com menor grau de urbanização, representam $12,62 \%$. Isso indica que nas cidades periféricas há maior demanda por gastos com saúde desse segmento populacional, que representa maiores custos para o sistema de saúde. Nas cidades periféricas também há uma menor cobertura de planos de saúde, e maior proporção de mulheres em relação ao número de homens, fatores que representam gastos adicionais. Há, ainda, uma proporção maior de domicílios com saneamento considerado inadequado, (14,63\% em comparação com 5,44\%), que também causa reflexos negativos na saúde geral da população.

Nas cidades centrais a pressão de custos se dá em razão da maior quantidade de médicos por mil habitantes. Por outro lado, nessas localidades há um ganho de escala em razão da menor quantidade de leitos por mil habitantes, uma vez que a taxa de ocupação dos leitos é maior.

A proporção de prefeitos pertencentes ao partido do governador e ao partido do presidente da república não apresenta uma distribuição significativamente diferente em relação à centralidade municipal.

\subsection{Dependência espacial}

Foi testada a dependência espacial utilizando matrizes de contiguidade Queen e Rook de primeira e segunda ordem, bem como as matrizes de distância de 30, 50 e 70 quilômetros. Para 
os gastos com saúde per capita e atenção básica per capita, independentemente da matriz de significância utilizada, todos os coeficientes de Moran mostraram-se diferentes de zero a 1\% de significância. Com relação à assistência hospitalar a dependência espacial é menos significativa, uma vez que há muitos municípios que não gastam com esse tipo de despesa. Entre as matrizes de significância testadas, apenas as de contiguidade de primeira ordem e a distância de 50 quilômetros resultaram em um I de Moran significativamente diferentes de zero a 5\% de significância (tabela 2).

Tabela 2

I de Moran

\begin{tabular}{|c|c|c|c|}
\hline & Saúde & Atenção Básica & $\begin{array}{l}\text { Assistência } \\
\text { Hospitalar }\end{array}$ \\
\hline \multirow{2}{*}{$\begin{array}{l}\text { Queen } 1 \\
\text { p-valor }\end{array}$} & 0,146 & 0,132 & 0,092 \\
\hline & 0,001 & 0,001 & 0,012 \\
\hline \multirow{2}{*}{$\begin{array}{l}\text { Queen2 } \\
\text { p-valor }\end{array}$} & 0,171 & 0,164 & 0,017 \\
\hline & 0,001 & 0,001 & 0,212 \\
\hline \multirow{2}{*}{$\begin{array}{l}\text { Rook } 1 \\
\text { p-valor }\end{array}$} & 0,150 & 0,136 & 0,008 \\
\hline & 0,002 & 0,001 & 0,020 \\
\hline \multirow{2}{*}{$\begin{array}{l}\text { Rook2 } \\
\text { P-valor }\end{array}$} & 0,166 & 0,157 & 0,018 \\
\hline & 0,001 & 0,001 & 0,221 \\
\hline \multirow{2}{*}{$\begin{array}{l}\text { Dist30 } \\
\text { p-valor }\end{array}$} & 0,190 & 0,186 & 0,027 \\
\hline & 0,001 & 0,001 & 0,138 \\
\hline \multirow{2}{*}{$\begin{array}{l}\text { Dist50 } \\
\text { p-valor }\end{array}$} & 0,163 & 0,168 & 0,036 \\
\hline & 0,001 & 0,001 & 0,030 \\
\hline \multirow{2}{*}{$\begin{array}{l}\text { Dist70 } \\
\text { p-valor }\end{array}$} & 0,170 & 0,162 & 0,019 \\
\hline & 0,001 & 0,001 & 0,057 \\
\hline
\end{tabular}

Fonte: Elaboração própria a partir dos dados coletados.

Procurou-se escolher uma mesma matriz para todas as quatro variáveis, a fim de que fosse possível comparar os resultados das regressões. Seguindo o critério de Baumont (2004), entre as três matrizes significativas, foi selecionada a de distância de 50 quilômetros, que mostrou maiores índices de Moran para todas as variáveis. Esta foi utilizada para estimação das regressões.

\subsection{Estimação da centralidade}

Preliminarmente à estimação das variáveis buscou-se identificar a estrutura de correlação entre as variáveis. Foram estimados os Fatores de Inflação da Variância (FIV) e nenhuma das 
variáveis apresentou FIV superior a 3, o que indica a inexistência de problema de multicolineariedade. Foi identificada uma correlação moderada entre a variável de estudo (centralidade) e a dependência financeira, optando-se por adicionar uma variável multiplicativa entre as duas (Cent*Depend) para isolar o efeito da dependência financeira dos municípios centrais nas variáveis dependentes e melhor compreender o efeito líquido da centralidade. Os resultados das regressões são apresentados na tabela 3 .

Tabela 3

\section{Regressões espaciais}

\begin{tabular}{|c|c|c|c|c|c|c|}
\hline & Saúde & $\begin{array}{l}\text { Atenção } \\
\text { Básica }\end{array}$ & $\begin{array}{l}\text { Assistência } \\
\text { Hospitalar }\end{array}$ & Saúde & $\begin{array}{l}\text { Atenção } \\
\text { Básica }\end{array}$ & $\begin{array}{r}\text { Assistência } \\
\text { Hospitalar }\end{array}$ \\
\hline \multirow{2}{*}{$\begin{array}{l}\text { W_saude } \\
\text { p-valor }\end{array}$} & 0.175 & 0.156 & 0.051 & 0.282 & 0.250 & 0.052 \\
\hline & 0.131 & 0.185 & 0.758 & 0.012 & 0.030 & 0.755 \\
\hline \multirow{2}{*}{$\begin{array}{l}\text { CONSTANT } \\
\text { p-valor }\end{array}$} & -311.454 & -414.194 & 43.656 & -388.915 & -303.270 & -80.336 \\
\hline & 0.155 & 0.075 & 0.694 & 0.085 & 0.209 & 0.490 \\
\hline \multirow{2}{*}{$\begin{array}{l}\text { PIB } \\
\text { p-valor }\end{array}$} & 0.003 & 0.002 & 0.001 & 0.003 & 0.002 & 0.001 \\
\hline & 0.002 & 0.041 & 0.077 & 0.000 & 0.066 & 0.014 \\
\hline \multirow{2}{*}{$\begin{array}{l}\text { Idosos } \\
\text { p-valor }\end{array}$} & 13.192 & 20.347 & -5.207 & 11.077 & 16.887 & -4.136 \\
\hline & 0.011 & 0.000 & 0.052 & 0.030 & 0.002 & 0.122 \\
\hline \multirow{2}{*}{$\begin{array}{l}\text { Crian } \\
\text { p-valor }\end{array}$} & -21.259 & -13.987 & -3.406 & -18.602 & -14.630 & -1.046 \\
\hline & 0.099 & 0.311 & 0.605 & 0.139 & 0.280 & 0.873 \\
\hline \multirow{2}{*}{$\begin{array}{l}\text { Gênero } \\
\text { p-valor }\end{array}$} & 1.854 & 1.364 & 0.606 & 1.699 & 0.592 & 1.070 \\
\hline & 0.200 & 0.382 & 0.415 & 0.236 & 0.703 & 0.153 \\
\hline \multirow{2}{*}{$\begin{array}{l}\text { Médicos } \\
\text { p-valor }\end{array}$} & 15.055 & 9.338 & 4.669 & 13.593 & 11.051 & 2.471 \\
\hline & 0.000 & 0.003 & 0.002 & 0.000 & 0.001 & 0.131 \\
\hline \multirow{2}{*}{$\begin{array}{l}\text { Leit } \\
\text { p-valor }\end{array}$} & -30.968 & -28.334 & -1.243 & -30.886 & -31.211 & 0.710 \\
\hline & 0.000 & 0.002 & 0.778 & 0.000 & 0.001 & 0.871 \\
\hline \multirow{2}{*}{$\begin{array}{l}\text { PlanSau } \\
\text { p-valor }\end{array}$} & 0.564 & -0.060 & 0.490 & 0.343 & 0.175 & 0.204 \\
\hline & 0.475 & 0.944 & 0.224 & 0.662 & 0.836 & 0.616 \\
\hline \multirow{2}{*}{$\begin{array}{l}\text { ESG } \\
\text { p-valor }\end{array}$} & 1.225 & 0.957 & 0.104 & 0.696 & 0.599 & 0.002 \\
\hline & 0.105 & 0.237 & 0.782 & 0.347 & 0.451 & 0.996 \\
\hline \multirow{2}{*}{$\begin{array}{l}\text { Mortal } \\
\text { p-valor }\end{array}$} & -10.776 & -13.874 & 3.028 & -9.785 & -12.196 & 2.460 \\
\hline & 0.100 & 0.048 & 0.369 & 0.124 & 0.076 & 0.458 \\
\hline \multirow{2}{*}{$\begin{array}{l}\text { Dgov } \\
\text { p-valor }\end{array}$} & -27.336 & -31.995 & 0.583 & -31.258 & -35.875 & 0.572 \\
\hline & 0.114 & 0.085 & 0.948 & 0.062 & 0.048 & 0.948 \\
\hline \multirow{2}{*}{$\begin{array}{l}\text { Dpres } \\
\text { p-valor }\end{array}$} & -36.979 & -43.759 & -0.951 & -30.309 & -34.015 & -3.686 \\
\hline & 0.167 & 0.128 & 0.945 & 0.244 & 0.227 & 0.787 \\
\hline \multirow{2}{*}{$\begin{array}{l}\text { Depen } \\
\text { p-valor }\end{array}$} & 416.738 & 512.328 & -82.279 & 499.733 & 479.261 & -7.631 \\
\hline & 0.000 & 0.000 & 0.082 & 0.000 & 0.000 & 0.885 \\
\hline
\end{tabular}




\begin{tabular}{|c|c|c|c|c|c|c|}
\hline & Saúde & $\begin{array}{l}\text { Atenção } \\
\text { Básica }\end{array}$ & $\begin{array}{l}\text { Assistência } \\
\text { Hospitalar }\end{array}$ & Saúde & $\begin{array}{l}\text { Atenção } \\
\text { Básica }\end{array}$ & $\begin{array}{l}\text { Assistência } \\
\text { Hospitalar }\end{array}$ \\
\hline \multirow{2}{*}{$\begin{array}{l}\text { PR } \\
\text { p-valor }\end{array}$} & 13.168 & 4.852 & -7.472 & 25.866 & 21.923 & -9.573 \\
\hline & 0.678 & 0.887 & 0.648 & 0.405 & 0.514 & 0.556 \\
\hline \multirow{2}{*}{$\begin{array}{l}\text { RS } \\
\text { p-valor }\end{array}$} & -12.575 & -17.164 & -0.581 & -6.020 & -12.026 & -0.159 \\
\hline & 0.638 & 0.551 & 0.966 & 0.817 & 0.669 & 0.991 \\
\hline \multirow{2}{*}{$\begin{array}{l}\text { ARG } \\
\text { p-valor }\end{array}$} & -39.388 & -41.971 & 1.687 & -15.822 & -22.072 & 1.955 \\
\hline & 0.382 & 0.387 & 0.942 & 0.718 & 0.642 & 0.932 \\
\hline \multirow{2}{*}{$\begin{array}{l}\text { Cent*Depend } \\
\text { p-valor }\end{array}$} & & & & -724.881 & -300.147 & -262.025 \\
\hline & & & & 0.000 & 0.181 & 0.015 \\
\hline \multirow{2}{*}{$\begin{array}{l}\text { CENTRALIDADE } \\
\text { p-valor }\end{array}$} & & & & 490.965 & 127.318 & 230.010 \\
\hline & & & & 0.003 & 0.469 & 0.007 \\
\hline R Quadrado & 0.39 & 0.39 & 0.11 & 0.42 & 0.42 & 0.14 \\
\hline Critério de Akaike & 3741.4 & 3783.6 & 3353.0 & 3727.5 & 3773.3 & 3347.4 \\
\hline Critério de Schwarz & 3804.0 & 3846.2 & 3415.5 & 3797.4 & 3843.3 & 3417.3 \\
\hline
\end{tabular}

Fonte: Elaboração própria a partir dos dados coletados.

\subsection{Discussão dos resultados}

Como esperado, a variável relativa à renda per capita mostrou-se significativa a 5\% para os gastos agregados de saúde e a 10\% para as duas subfunções, apresentando sinal positivo em todos os casos, confirmando Freeman (2003).

A proporção de pessoas com mais de 65 anos na população também apresentou sinal positivo em todas as regressões, confirmando as evidências encontradas em Di Matteo (2005). No entanto, mostrou-se significativamente diferente de zero, a 5\% de significância, apenas para os gastos com saúde per capita e atenção básica per capita. Para a variável assistência hospitalar per capita ela é significativa somente a 10\%, sem considerar a centralidade, e não é significativa ao se incorporar a centralidade ao modelo.

Essas evidências são coerentes com a teoria dos lugares centrais, pois os hospitais atendem à população idosa do município e de seus vizinhos, o que faz com que a proporção de idosos do município não represente com tanta fidedignidade a população de idosos atendida pela rede hospitalar. Já os gastos com atenção básica, por serem direcionados a serviços de menor complexidade, disponíveis em quase todos os municípios, são mais sensíveis à estrutura etária da população local.

A proporção de crianças na população, por outro lado, apresentou sinal negativo, contrariando a literatura. Os resultados, porém, não são significativos a 5\% de significância. As variáveis relativas a gênero, mortalidade e cobertura do plano de saúde, tal como nos estudos de Moscone e Knapp (2005); Moscone, Knapp e Tosetti (2007) e Novaes e Mattos (2010), não se mostraram consistentemente significativas. 
O número de profissionais de saúde por mil habitantes apresentou sinal positivo, mostrando que, quanto maior a proporção de médicos, tanto maiores são os gastos per capita na função saúde, assim como previsto por Costa-Font e Moscone (2008). A variável de leitos por mil habitantes apresentou sinal negativo, evidenciando um ganho de escala, também como esperado, confirmando Giannoni e Hitiris (2002) e Costa-Font e Pons-Novell (2007). As duas variáveis apresentaram-se significativas a 5\% para gastos com saúde per capita e gastos com atenção básica per capita. Para os gastos com assistência hospitalar, apesar dos sinais corretos das variáveis, não se mostraram significativas. A proporção de domicílios apresentando condições inadequadas de esgotamento sanitário não se mostrou significativa.

Com relação às dummies utilizadas para diferenciar os gastos dos municípios que pertencem ao partido do governador e do presidente da república, ambas apresentam sinal negativo, indicando um efeito substituição do gasto municipal pelo gasto federal e estadual. Essas variáveis, no entanto, não resultaram significativas. Essas evidências, portanto, não suportam Novaes e Mattos (2010).

A dependência financeira, por outro lado, mostrou-se significativamente positiva em relação às de gastos com saúde e atenção básica. Essa relação pode ser explicada considerando-se que a maior dependência faz com que aumente a sujeição do município em relação às políticas estaduais e federais. Por outro lado, a interação da dependência com a centralidade mostra que para os municípios centrais o efeito é inverso, indicando que municípios centrais mais dependentes investem proporcionalmente menos do que os centrais não dependentes. A variável de centralidade mostrou-se significativamente diferente de zero a $1 \%$ de significância para os modelos de gastos com saúde per capita e assistência hospitalar per capita, apresentando sinal positivo para ambos. Isso indica que os municípios centrais apresentam valores maiores de gastos com saúde per capita, assistência hospitalar per capita, confirmando a hipótese 2. Essas evidências estão coerentes com a teoria uma vez que se espera que os municípios centrais apresentem maiores gastos médicos com despesas hospitalares, em razão do atendimento tanto à sua população como à população de seus vizinhos. Já no modelo de gastos com atenção básica não foi significativamente diferente de zero, não podendo ser aceita a hipótese 1 de que há diferença entre gastos de municípios centrais e não centrais nessa função.

A inclusão da variável de centralidade fez com que os coeficientes de dependência horizontal aumentassem em todos os modelos (tabela 4), confirmando a hipótese 3 da pesquisa e o trabalho de Revelli (2003).

Tabela 4

Coeficientes de dependência espacial

\begin{tabular}{|lcc|}
\hline & Sem centralidade & Com centralidade \\
\cline { 2 - 3 } Saúde & 0.175 & 0.282 \\
Atenção Básica & 0.156 & 0.250 \\
Assistência Hospitalar & 0.051 & 0.052 \\
Saúde Líquido & 0.240 & 0.334 \\
\hline
\end{tabular}

Fonte: Elaboração própria a partir dos dados coletados. 
Isso acontece porque os coeficientes de dependência nos modelos sem centralidade capturam o efeito médio, tanto da interação positiva quanto da negativa. Já com a inclusão da variável de centralidade, que é uma proxy da interação vertical entre municípios, parte do efeito negativo é capturado, fazendo com que a interação positiva da interação vertical fique mais bem evidenciada. A síntese dos testes de hipótese é apresentada no quadro 3.

Quadro 3

Síntese das hipóteses

\begin{tabular}{|lc|}
\hline \multicolumn{1}{|c|}{ Hipóteses } & Situação \\
\hline (H1): A centralidade está negativamente associada ao gasto público em Atenção Básica; & Rejeitada \\
(H2): A centralidade está positivamente associada ao gasto público per capita em Assistência Hospitalar e & Confirmada \\
Ambulatorial; & Confirmada \\
(H3): O coeficiente de dependência espacial aumenta com a inclusão da variável de centralidade. & \\
\hline
\end{tabular}

Fonte: Elaboração própria.

\section{Considerações finais}

O presente estudo procurou testar a influência da centralidade no padrão de interdependência horizontal nos gastos públicos de saúde. A partir da teoria dos lugares centrais foi proposta a existência de uma interação vertical entre municípios centrais e periféricos. Pressupõe-se que a interação vertical acontece simultaneamente à interação horizontal, atenuando seu efeito, uma vez que as duas atuam em sentidos opostos. Para testar essa hipótese foram especificados modelos sem considerar a centralidade e modelos que incorporaram a centralidade por meio de uma dummy para diferenciar os gastos dos municípios centrais dos periféricos. Adicionalmente foram testados os principais determinantes dos gastos públicos em saúde trazidos pela literatura.

Verificou-se que o padrão de gasto das localidades centrais apresenta diferença em relação ao padrão de gastos das localidades periféricas, principalmente no que diz respeito aos gastos com assistência hospitalar. Os gastos hospitalares são mais influenciados pela interação vertical do que horizontal, ao passo que os gastos com atenção básica parecem não sofrer da dependência vertical e apresentam uma dependência horizontal mais pronunciada, confirmando Costa-Font e Moscone (2008).

Ainda constatou-se que a força da interação horizontal é intensificada quando se inclui a variável de centralidade, o que indica que tal variável consegue capturar parte da interação negativa da interação vertical, corroborando Revelli (2003) e Werck, Heyndels e Geys (2008).

Esses resultados indicam que a consideração da centralidade é fundamental para a compreensão dos padrões de interdependência espacial, respondendo a pergunta de pesquisa proposta. 
Também são levantadas questões importantes a serem consideradas no desenho de políticas públicas, tais como:

、 Localidades centrais possuem um padrão de gasto diferente em relação às localidades periféricas, principalmente no que diz respeito a gastos com assistência hospitalar, uma vez que há um atendimento inter-regional. Isso implica a necessidade de políticas compensatórias para esse maior volume de gastos;

- Quanto maior a dependência dos municípios centrais em relação às transferências de outras esferas, menores serão seus gastos globais em saúde, principalmente no que diz respeito aos gastos com assistência hospitalar. Isso implica que o aumento de investimentos em saúde por parte dos municípios centrais é função de políticas públicas que lhes confiram maior autonomia financeira;

、 Nos municípios periféricos, quanto maior a dependência, maiores são os gastos com saúde. Portanto, o investimento em saúde será maior quanto menor autonomia financeira for concedida aos entes periféricos;

v A dependência vertical em relação a outros municípios nos gastos com atenção básica é praticamente inexistente, contudo, a relação horizontal é muito forte, indicando a existência de um efeito de yardstick competition nessa modadalidade. Esse padrão sugere a existência de gastos possivelmente direcionados para fins eleitorais.

Por fim, cabe ressaltar que o presente trabalho apresenta limitações geográficas, por utilizar dados apenas de municípios catarinenses; temporais, por trabalhar apenas com o ano de 2010 sem expandir para um período maior; e metodológica, por optar pela utilização de um modelo de gasto que não discrimina a fonte de recurso. Recomenda-se para trabalhos futuros a exploração desses pontos.

\section{Referências}

ALMEIDA, Eduardo. Econometria espacial aplicada. Campinas: Alínea, 2012.

ARONSSON, Thomas; LUNDBERG, Johan; WIKSTRÖM, Magnus. The impact of regional public expenditures on the local decision to spend. Regional Science and Urban Economics, v. 30, n. 2, p. 185-202, 2000. Disponível em: <www.sciencedirect.com/science/article/pii/S016604629900040X >. Acesso em: 13 dez. 2014.

BAUMONT, Catherine. Spatial effects in housing price models: do housing prices acpitalize urban development policies in the agglomeration of Dijon (1999)? Université de Bourgogne, 2004.

BESLEY, Timothy; CASE, Anne. Incumbent behavior: vote-seeking, tax-setting, and yardstick competition. The American Economic Review, v. 85, n. 1, p. 25-45, 1995. Disponível em: <www.jstor. org/stable/2117994>. Acesso em: 13 dez. 2014. 
BOULDING, Carew; BROWN, David S. Political competition and local social spending: evidence from Brazil. Studies in Comparative International Development, v. 49, n. 2, p. 197-216, 2014. Disponível em: <http://dx.doi.org/10.1007/s12116-013-9145-8>. Acesso em: 13 dez. 2014.

BRUECKNER, Jan K. Strategic interaction among governments: an overview of empirical studies. International Regional Science Review, v. 26, n. 2, p. 175-188, 2003. Disponível em: <www.scopus. com/inward/record.url? eid=2-s2.0-0037391333\&partnerID=40\&md5=bc4c55f6e26488f2a16b4e6e436402cb>. Acesso em: 13 dez. 2014.

CASE, Anne C.; ROSEN, Harvey S.; HINES JR., James R. Budget spillovers and fiscal policy interdependence: evidence from the states. Journal of Public Economics, v. 52, n. 3, p. 285-307, 1993. Disponível em: <www.sciencedirect.com/science/article/pii/004727279390036S>. Acesso em: 13 dez. 2014.

CHRISTALLER, Walter. Central places in southern Germany. Englewood Cliffs: Prentice-Hall, 1966. CONASS. Conselho Nacional de Secretários de Saúde. O financiamento da saúde. Brasília: Conass, 2011.

COSTA-FONT, Joan; MOSCONE, Francesco. The impact of decentralization and inter-territorial interactions on Spanish health expenditure. Empirical Economics, v. 34, n. 1, p. 167-184, 2008. Disponível em: <http://dx.doi.org/10.1007/s00181-007-0166-x>. Acesso em: 13 dez. 2014.

COSTA-FONT, Joan; PONS-NOVELL, Jordi. Public health expenditure and spatial interactions in a decentralized national health system. Health Economics, v. 16, n. 3, p. 291-306, 2007. Disponível em: <http://dx.doi.org/10.1002/hec.1154>. Acesso em: 13 dez. 2014.

DI MATTEO, Livio. The macro determinants of health expenditure in the United States and Canada: assessing the impact of income, age distribution and time. Health Policy, v. 71, n. 1, p. 23-42, 2005. Disponível em: <www.sciencedirect.com/science/article/pii/S0168851004001009>. Acesso em: 13 dez. 2014.

ELLIS, Randall P.; MCGUIRE, Thomas G. Hospital response to prospective payment: moral hazard, selection, and practice-style effects. Journal of Health Economics, v. 15, n. 3, p. 257-277, 1996. Disponível em: <www.sciencedirect.com/science/article/pii/0167629696000021>. Acesso em: 13 dez. 2014.

FREEMAN, Donald G. Is health care a necessity or a luxury? Pooled estimates of income elasticity from US state-level data. Applied Economics, v. 35, n. 5, p. 495-502, 2003. Disponível em: <http:// dx.doi.org/10.1080/00036840210138374>. Acesso em: 13 dez. 2014.

GIANNONI, Margherita; HITIRIS, Theodore. The regional impact of health care expenditure: the case of Italy. Applied Economics, v. 34, n. 14, p. 1829-1836, 2002. Disponível em: <www.tandfonline.com/doi/abs/10.1080/00036840210126809>. Acesso em: 13 dez. 2014.

ISEN, Adam. Do local government fiscal spillovers exist? Evidence from counties, municipalities, and school districts. Journal of Public Economics, v. 110, p. 57-73, 2014. Disponível em: <www. sciencedirect.com/science/article/pii/S0047272713002478>. Acesso em: 13 dez. 2014. 
KELEJIAN, Harry; ROBINSON, Dennis P. A suggested method of estimation for spatial interdependent models with autocorrelated errors, and an application to a county expenditure model. Papers in Regional Science, v. 72, n. 3, p. 297-312, 1993. Disponível em: <http://dx.doi.org/10.1007/ BF01434278>. Acesso em: 13 dez. 2014.

LE MAUX, Benoît; ROCABOY, Yvon; GOODSPEED, Timothy. Political fragmentation, party ideology and public expenditures. Public Choice, v. 147, n. 1-2, p. 43-67, 2011. Disponível em: <http:// dx.doi.org/10.1007/s11127-010-9603-z>. Acesso em: 13 dez. 2014.

LÖSCH, August. The economics of location. New Haven: Yale University, 1954.

MENDES, Áquilas N.; SANTOS, Suzana B. D. S. Financiamento descentralizado da saúde: a contribuição dos municípios paulistas. Saúde e Sociedade, v. 9, n. 1-2, p. 111-125, 2000. Disponível em: $<$ www.scielo.br/scielo.php?script =sci_arttext\&pid=S0104-12902000000100009\&nrm $=$ iso $>$. Acesso em: 13 dez. 2014.

MOSCONE, Francesco; KNAPP, Martin. Exploring the spatial pattern of mental health expenditure. Journal of Mental Health Policy and Economics, v. 8, n. 4, p. 205-217, 2005. Disponível em: <www. scopus.com/inward/record.url?eid=2-s2.0-31344478568\&partnerID =40\&md5=4020731 add0091c708a01d53dd79f07e>. Acesso em: 13 dez. 2014.

MOSCONE, Francesco; KNAPP, Martin; TOSETTI, Elisa. Mental health expenditure in England: A spatial panel approach. Journal of Health Economics, v. 26, n. 4, p. 842-864, 2007. Disponível em: <www.sciencedirect.com/science/article/pii/S0167629606001445>. Acesso em: 13 dez. 2014.

MURDOCH, James C.; RAHMATIAN, Morteza; THAYER, Mark A. A spatially autoregressive median voter model of recreation expenditures. Public Finance Quarterly, v. 21, n. 3, p. 334-350, jul. 1993. Disponível em: <<Go to ISI>://WOS:A1993LH60800006>. Acesso em: 13 dez. 2014.

NAKAMURA, Daisuke. Social participation and social capital with equity and efficiency: An approach from central-place theory. Applied Geography, v. 49, p. 54-57, 2014. Disponível em: <www.sciencedirect.com/science/article/pii/S0143622813002130>. Acesso em: 13 dez. 2014.

NOVAES, Lucas; MATTOS, Enlinson. O efeito da intenção de reeleição sobre gastos em saúde: uma análise com base no modelo de reputação política. Revista de Economia Política, v. 30, p. 140-158, 2010. Disponível em: <www.scielo.br/scielo.php?script=sci_arttext\&pi$\mathrm{d}=$ S0101-31572010000100009\&nrm=iso > . Acesso em: $13 \mathrm{dez} .2014$.

POTRAFKE, Niklas. Did globalization restrict partisan politics? An empirical evaluation of social expenditures in a panel of OECD countries. Public Choice, v. 140, n. 1-2, p. 105-124, 2009. Disponível em: <http://dx.doi.org/10.1007/s11127-009-9414-2>. Acesso em: 13 dez. 2014.

PRIETO, David C.; LAGO-PEÑAS, Santiago. Decomposing the determinants of health care expenditure: the case of Spain. The European Journal of Health Economics, v. 13, n. 1, p. 19-27, 2012. Disponível em: <http://dx.doi.org/10.1007/s10198-010-0276-9>. Acesso em: 13 dez. 2014.

REVELLI, Federico. On spatial public finance empirics. International Tax and Public Finance, v. 12, n. 4, p. 475-492, 2005. Disponível em: < http://dx.doi.org/10.1007/s10797-005-4199-9>. Acesso em: 13 dez. 2014. 
REVELLI, Federico. Reaction or interaction? Spatial process identification in multi-tiered government structures. Journal of Urban Economics, v. 53, n. 1, p. 29-53, 2003. Disponível em: <www. sciencedirect.com/science/article/pii/S0094119002005120>. Acesso em: 13 dez. 2014.

SOLÉ-OLLÉ, Albert. Expenditure spillovers and fiscal interactions: Empirical evidence from local governments in Spain. Journal of Urban Economics, v. 59, n. 1, p. 32-53, 2006. Disponível em: <www.sciencedirect.com/science/article/pii/S0094119005000604>. Acesso em: 13 dez. 2014.

SOUSA, Kleber. M. Efeitos da arrecadação tributária e dos gastos públicos em serviços de saúde dos municípios de Pernambuco. Dissertação (mestrado) — Escola Brasileira de Administração Pública e de Empresas, Centro de Formação Acadêmica e Pesquisa, Fundação Getulio Vargas, Rio de Janeiro, 2012.

UGÁ, Maria A. D. Sistemas de alocação de recursos a prestadores de serviços de saúde - a experiência internacional. Ciência \& Saúde Coletiva, v. 17, n. 12, p. 3437-3445, 2012. Disponível em: $<$ www.scielo.br/scielo.php?script=sci_arttext\&pid=S1413-81232012001200028\&nrm =iso > . Acesso em: 13 dez. 2014.

VAZQUEZ, Daniel A. Efeitos da regulação federal sobre o financiamento da saúde. Cadernos de Saúde Pública, v. 27, n. 6, p. 1201-1212, 2011. Disponível em: <www.scielo.br/scielo.php?scrip$\mathrm{t}=\mathrm{sci}$ _arttext\&pid=S0102-311X2011000600017\&nrm=iso >. Acesso em: 13 dez. 2014.

WERCK, Kristien; HEYNDELS, Bruno; GEYS, Benny. The impact of 'central places' on spatial spending patterns: evidence from Flemish local government cultural expenditures. Journal of Cultural Economics, v. 32, n. 1, p. 35-58, 2008. Disponível em: <http://dx.doi.org/10.1007/s10824-0079056-5>. Acesso em: 13 dez. 2014.

WILSON, John D. Theories of tax competition. National Tax Journal, v. 52, n. 2, p. 269-304, 1999. Disponível em: <www.socsci.uci.edu/ jkbrueck/course\%20readings/Econ\%20272B\%20readings/ wilson\%20ntj.pdf>. Acesso em: 13 dez. 2014.

Robson Fernandes Soares é doutorando em administração na Universidade Positivo (UP). E-mail: robsoares88@gmail.com.

Ademir Clemente é doutor em engenharia de transporte e professor da Universidade Federal do Paraná (UFPR). E-mail: ademir@ufpr.br.

Fátima de Souza Freire é doutora em economia e professora da Universidade de Brasília (UnB). E-mail: ffreire@unb.br.

Jorge Eduardo Scarpin é doutor em controladoria e contabilidade e professor da Universidade Federal do Paraná (UFPR). E-mail: jscarpin@gmail.com. 